\title{
YACHACHIY: LA AUTOEVALUACIÓN INSTITUCIONAL PROPEDEÚTICA DE CALIDAD EDUCATIVA
}

\section{YACHACHIY: THE PRO-ETHICAL INSTITUTIONAL SELF-ASSESSMENT OF EDUCATIONAL QUALITY}

\section{Mileydi Flores Fernández ${ }^{1}$}

\section{Elmer Llanos Díaz}

\author{
Alfredo Puican Carreño ${ }^{3}$ \\ Universidad Cesar Vallejo Lambayeque - Perú
}

\section{RESUMEN}

La publicación está dirigida a promover autoevaluación institucional con fines de acreditación en las instituciones educativas que brindan Educación Básica Regular. Esta pone en el tablón las limitaciones del escenario; escasos avances en los factores de gestión referidos a la dirección de la institución, el apoyo permanente al docente, la incorporación de las familias y comunidad al trabajo educativo, la gestión de la información, implementación de recursos e in-

\section{$1 \quad$ Mifloresf@Ucvvirtual.Edu.Pe}

Teléfono Celular 954649935 Investigador Universidad Cesar Vallejo Lambayeque - Perú Mileydi Flores Fernández 0000 0002-6257-1795

\section{$2 \quad$ Ellanosd@Ucvvirtual.Edu.Pe}

Teléfono Celular 920297375 Investigador Universidad Cesar Vallejo Lambayeque - Perú Elmer Llanos Díaz 0000-0002$\underline{6955-8219}$

3 Apuican@Unprg.Edu.Pe

Teléfono Celular 979662192 Investigador Universidad Cesar Vallejo Lambayeque - Perú Alfredo Puican Carreño 0000$\underline{0002-1262-1985}$ fraestructura adecuada para el aprendizaje, con una mirada sistemática y que, en muchos casos, explica las consecuencias que derivan en exclusión de estudiantes dada la condición cultural o social, acompañado de un entendimiento errado y punitivo de la autoevaluación, aún instalada en el imaginario de la comunidad. Esta situación trae como consecuencia bajos niveles de percepción sobre las limitaciones en los factores, estándares e indicadores de desarrollo institucional; por ese motivo, se debe incorporar el proceso de autoevaluación como parte de la gestión institucional con miras a desarrollar enfoques que nutran la gestión en torno a una mejor comprensión de la realidad y su oportuna toma de decisiones en la priorización de la problemática, así como en elaboración de planes de mejora, cimentando un ambiente de sano compromiso de, con y para la comunidad con una 
mirada integral orientada sinérgicamente a la mejora de la calidad educativa.

\section{PALABRAS CLAVE:}

Autoevaluación institucional, trabajo con familias, soporte pedagógico a docentes, infraestructura educativa

\section{KEY WORDS:}

Institutional self-evaluation, work with families, pedagogical support to teachers, educational infrastructure.

\section{INTRODUCCIÓN}

La educación básica no ha cumplido con desarrollar las competencias de manera integral del estudiante, que implican consolidar sus potencialidades, capacidades y valores, así como fortalecer sus actitudes que le permitan desenvolverse de manera eficaz en el ámbito socio cultural. La Unesco ha dejado claramente definido que, si un sistema educativo se adecúa a las necesidades de las personas, garantiza la equidad en oportunidades para concretizar sus derechos y, finalmente, la igualdad y la democracia. En ese sentido, las políticas educativas en Perú buscan implementar una educación de calidad, evitando la deserción y promoviendo la inserción oportuna y progresiva en igualdad de condiciones; más aún cuando hablamos de demandas diversas que exigen implementar gestiones con enfoque intercultural, donde sea posible el mutuo aprendizaje, optimización en el uso de recursos y la construcción de convivencia armoniosa. Sin embargo, para llegar a ello, es importante intervenir en las primeras organizaciones que tienen contacto con la persona. Me refiero a las instituciones educativas que, valgan verdades, a la fecha no han desarrollado la cultura de la autoevaluación institucional, situación que no permite advertir las limitaciones que impiden formar integralmente a las personas, lo que dimensiona los enfoques filosóficos, científico multidisciplinar y pragmático. Para hacer ese seguimiento, la Oficina de Medición de la Calidad implementó la evaluación censal en las áreas de Comprensión Lectora y Matemática, los resultados en la última evaluación del año 2019 fueron desalentadores.

Según el Reporte Nacional de Evaluación Censal 2019 MINEDU, nos muestran que, en Comprensión Lectora de $2 .^{\circ}$ grado de primaria, el mayor porcentaje de estudiantes se encuentra en inicio; así, un $3.1 \%$ está en nivel de inicio, $60.3 \%$ en proceso y solo $36.7 \%$ logró la competencia de comprensión lectora en la región Lambayeque; de la misma fuente, en Matemática, 53.8\% está en nivel de inicio, 31.6 $\%$ en proceso y tan solo el $14.6 \%$ consiguió el desarrollo de la competencia matemática. Estos resultados evidencian que no ha sido suficiente la gestión hasta el día de hoy. «La autoevaluación no solo implica aplicar una serie de procedimientos (recoger, presentar, procesar datos, entre otros). Es también un proceso continuo de interpretación y negociación» (Palma, 2001, p. 4). «En este modelo de autoevaluación de tipo participativo, donde impera el diálogo, es posible generar consensos que permitan cumplir los fines de la institución» (Alaiz et al., 2003, p. 61). Dicho esto, si los procesos de autoevaluación de estándares no son eficaces, tampoco el sistema educativo lo será. Por ejemplo, es evidente el escaso soporte al desempeño docente; no obstante, Lea F. Vezub (2007) manifiesta que los mecanismos implementados en la actualidad obedecen a una intencionalidad política dirigidos por la lógica del mercado, la competitividad y no el bienestar común que es el fin supremo de la cosa pública, además persiste el temor de la comunidad de docentes, ya que hay una tendencia a ser interpelados en su actuación como protagonistas activos y reflexivos, o como mediadores de lo planificado; así, este es el aliado estratégico para la transformación educativa. Otros estándares son la vinculación en el trabajo 
con familias y comunidad, el uso adecuado de la información, la infraestructura y recursos de aprendizaje que son indicadores del proceso de autoevaluación y puntos de partida para planear las mejoras.

La propuesta de acreditación del Sistema Nacional de Evaluación y Acreditación Educativa (Sineace) en Perú promulgada por Ley $\mathrm{N}^{\circ}{ }^{\circ} 28740$, aprobado por decreto supremo N. ${ }^{\circ}$ 018-2007-ED, asume mecanismos para mejorar la calidad educativa, apostando porque el proceso de acreditación garantice la entrega del servicio educativo de calidad y el reconocimiento público a las instituciones educativas por su gestión óptima. Sin embargo, en la actualidad, se puede entrever que existe aún una débil cultura autoevaluativa en las instituciones educativas, pero que es un buen talante para reconocer las falencias que permitan apuntalar las limitaciones a fortalecerse, determinando la finalidad institucional en función a la pertinencia con la sociedad.

El proceso de autoevaluación visto desde la Ley General de Educación de Perú N. ${ }^{\circ} 28044$ prescribe la integralidad en la formación del educando físicamente, afectivamente y cognitivamente. De este modo, construye su identidad personal y social, promoviendo el ejercicio de una ciudadanía responsable que le permita insertarse al mercado laboral y dinamizar su economía en función de su proyecto de vida. Para ello la persona incorpora capacidades, valores y actitudes que le sirven de andamiaje para sostener su aprendizaje en diversos campos como ciencias, humanidades, técnica, cultura, arte, educación física y deportes a lo largo de la vida, adicionando habilidades de convivencia e interacción en la sociedad del conocimiento, como es el uso de las tecnologías de información y comunicación. Sumando al proyecto nacional, para lograrlo, es imprescindible hacer la autoevaluación institu- cional, que implica una revisión de los procedimientos y los productos entregados hasta el momento en la organización escolar, buscando incorporar el aporte de todos los actores del proceso educativo y garantizar la pertinencia de la oferta formativa que la sociedad requiere. Asimismo, en la práctica, la metodología de trabajo de consenso recoge el interés y aporte de los miembros de la comunidad, así como su compromiso en busca de la mejora para la gestión.

La autoevaluación institucional desde el Proyecto Educativo Nacional al 2036 (PEN) tiene dos enfoques en su visión: el primero es un enfoque humanista que se sustenta en generar condiciones para que los peruanos nos desarrollemos y prosperemos a lo largo de nuestras vidas contribuyendo al proyecto país; el segundo es el enfoque de derecho que tiene que ver con el reconocimiento de la ciudadanía y el quehacer ciudadano basados en el ejercicio de la libertad, la convivencia, diálogo intergeneracional e intercultural, construcción de la democracia equitativa e inclusiva con responsabilidad ambiental. Para lograrlo, determina 5 propósitos: uso universal e intensivo de tecnologías digitales, fortalecer el carácter público de la educación, poniendo especial atención en las expectativas de las personas, adecuado uso del financiamiento, gestión autónoma de las instituciones educativas articuladas a otras instituciones e instancias descentralizadas, evitando la violencia, segregación y discriminación. Es precisamente en este punto que vamos a acentuar la íes, ya que esta situación exige una gestión que empiece desde y para dentro enmarcados con una efectiva autoevaluación que identifique claramente las demandas y potencialidades.

El Acuerdo Nacional fue diseñado y firmado en el año 2002 por el Gobierno. En este, estuvieron involucrados representantes de la sociedad civil y los partidos políticos, sistematiza las expectativas de los miembros de la sociedad por desarrollar la educación. En la política $\mathrm{N}$. $^{\circ}$ 
12, explícitamente, manifiesta la significación del acompañamiento y la universalización de la educación de calidad; para ello, considera determinante evaluar el uso del presupuesto redireccionando prioridades, generando condiciones óptimas a los estudiantes. Asimismo, considera que las brechas de inequidad educativa deben ser cubiertas, implementando mecanismos de certificación y calificación que desarrollen la cultura de autoevaluación y vigilancia, aumentando la exigencia en la educación pública y privada.

La Política Nacional de Competitividad y Productividad destaca a la educación como función clave del desarrollo nacional, ya que mediante ella se cumple el objetivo prioritario de competitividad como es fortalecer las capacidades de la fuerza laboral (oferta formativa) y articularlas con los requerimientos del mercado laboral. De este modo, se garantiza el acceso y la calidad de educación superior; asimismo, el acceso de la población en edad escolar a recibir servicios públicos de calidad, consolidar la reforma magisterial en busca de asegurar el fortalecimiento del capital humano. Todo ello a partir de desarrollar y certificar sus competencias laborales, logrando su participación en un mercado dinámico y eficiente, por lo que será imprescindible la mejora de la gestión del sistema educativo basados en la autoevaluación continua y toma de decisiones oportunas.

Con esta publicación tenemos la intención de promover el proceso de autoevaluación institucional en Educación Básica Regular como base para recoger información de manera sistemática sobre estándares de la gestión institucional. Su finalidad es reconocer oportunamente las fortalezas, oportunidades, debilidades y amenazas, así como proponer planes de mejora para superar los nimios avances en los estándares de calidad, que se evidencian en la exclusión, pobres niveles de desempeño de los estudiantes. Situación motivada muchas veces por una comprensión punitiva de la autoevaluación de la gestión, aún instalada en el imaginario de la comunidad educativa, en torno a su particular comprensión sobre la construcción de la calidad educativa.

\section{METODOLOGÍA}

La presente investigación cualitativa aplicada a una institución educativa básica regular es descriptiva-propositiva, ya que se realizó una autoevaluación de los procesos de gestión de la calidad de la I. E., y propositiva porque, a partir de los hallazgos, es plausible de proponer planes de mejora. Su base epistemológica es la teoría sistémica; en la medida en que se va a construir y producir información relevante y modelar la construcción y producción del saber científico, respecto a la optimización de la gestión de modo integral.

La población son todas la I. E. de formación básica regular de la región Lambayeque y la muestra fue un centro educativo de gestión pública con selección de tipo no probabilística, seleccionada con criterio de conveniencia, debido a que es la primera institución educativa pública que ha iniciado el proceso de autoevaluación para la acreditación en la región. Esta modalidad "considera la muestra como unidad social como universo de investigación y observación» (Pérez; 1994: I-87). Los resultados son útiles para el centro educativo donde se aplicó la autoevaluación; y sirve de referencia para otras instituciones de educación básica regular. Además, usamos la técnica encuesta y el instrumento ficha de encuesta, a través de la utilización de cuestionarios, fichas de análisis documental y la técnica de análisis documental. Se utilizó el software del modelo de autoevaluación de la calidad de gestión educativa con fines de acreditación del Ipeba.

También se utilizó la media aritmética para el análisis y discusión de los resultados: 
La media aritmética es el valor obtenido al sumar todos los datos y dividir el resultado entre el número total de datos.

$$
x^{-} X_{1+} X_{2}+X_{3}+\ldots+X_{n}
$$

$\mathrm{N}$

Dónde: $X$ es el símbolo de la media aritmética.

Sobre el método de análisis de datos también se usaron las matrices de priorización de problemas. Asimismo, se emplearon la espina de Ishikawa y se implementó pentágono relacional de factores en el análisis de resultados para analizar las causas y las posibles soluciones, recogiendo información relevante utilizada únicamente para efectos de la presente investigación.

\section{RESULTADOS}

Los resultados muestran que en la primera etapa los miembros de la institución educativa dan un salto cualitativo importante, ya que deciden autoevaluar la gestión y buscan el diagnóstico en concordancia a los estandares previstos. La selección de la comisión de autoevaluación se llevó a cabo de manera democrática contando con los miembros de la institución educativa, que para este caso fueron: 440 estudiantes en ambos turnos, 2 directivos, 31 docentes, 3 auxiliares de educación y 7 personal administrativo y servicio. Las matrices están estructuradas en 5 factores; que son aspectos esenciales en el desarrollo de los aprendizajes de los estudiantes, como son: la dirección institucional, el soporte al desempeño docente, el trabajo conjunto con las familias y la comunidad, el uso de la información, la infraestructura y los recursos para el aprendizaje. Además, estos factores están conformados por estándares que precisan los logros esperados para cada factor, son 12 y 43 indicadores que determinan el nivel logro verificable de cada estándar.

\section{Nivel de calidad educativa por factor en la Institución Educativa Santo Toribio de Mogrovejo de Zaña.}

Figura 1. Results obtained with the Microsoft Excel program

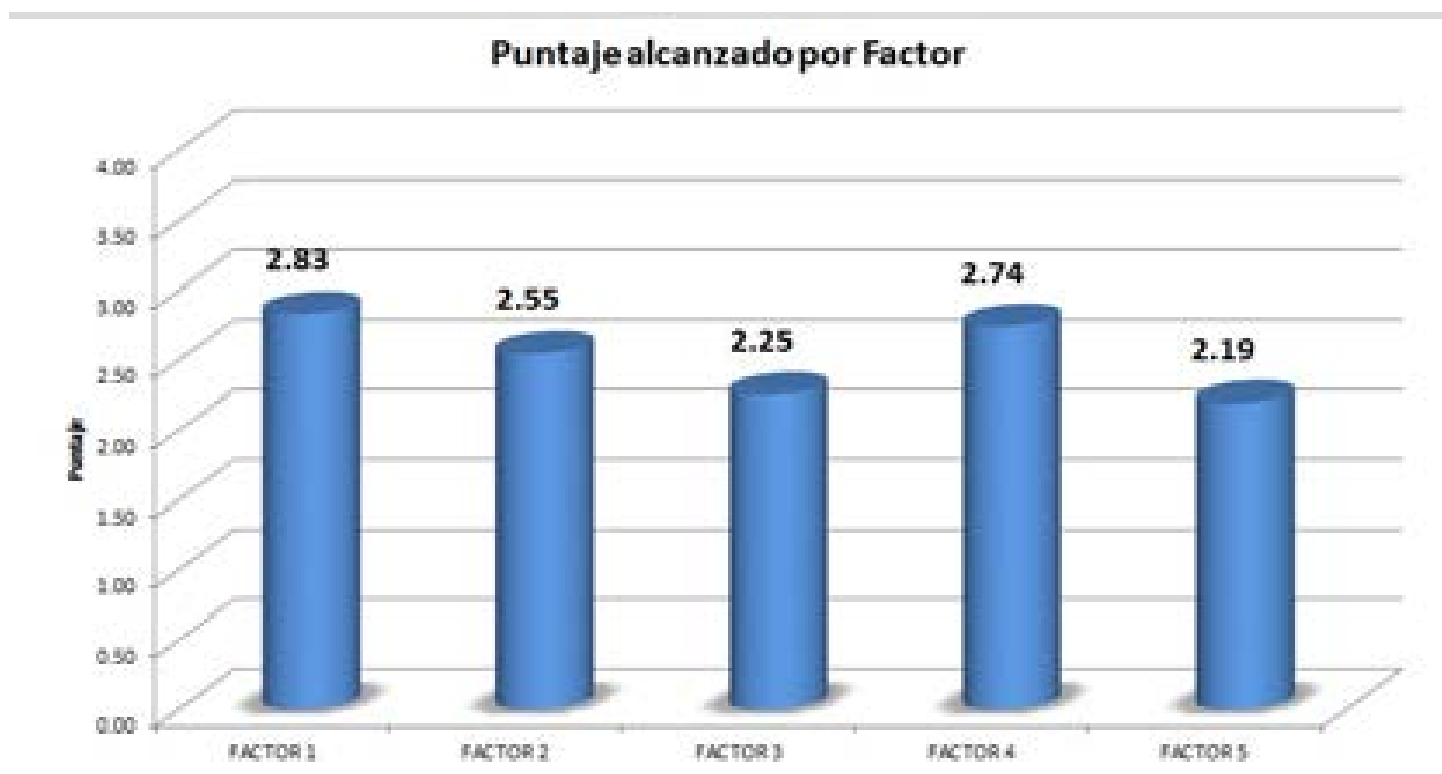

Elaboración propia. 


\section{Nivel de calidad educativa por estándar en la Institución Educativa Santo Toribio de Mogrovejo de Zaña}

Figura 2. Resultados obtenidos por el programa Excel

\section{Puntaje alcanzado por Estándar}

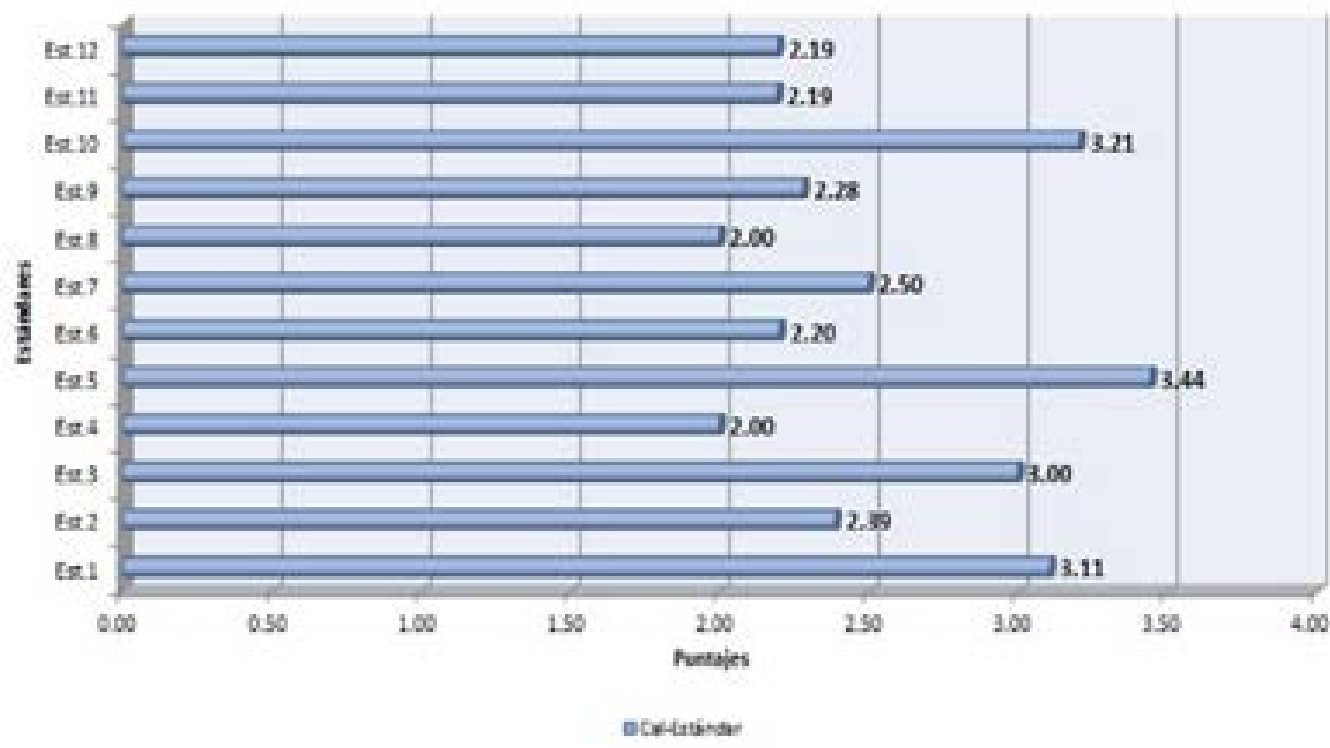

Elaboración propia.

Según la información recogida por los instrumentos llegamos a las siguientes precisiones:

Tabla 1

Nivel de avance de logro de estándares en la autoevaluación

\begin{tabular}{|c|c|c|}
\hline Factor & Estándares & Calificativo \\
\hline \multirow{3}{*}{ 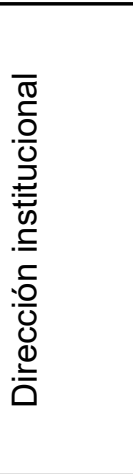 } & $\begin{array}{l}\text { 1. Participación en la construcción del proyecto educativo enfocado } \\
\text { en la mejora del proceso de enseñanza-aprendizaje de manera per- } \\
\text { tinente, inclusiva. }\end{array}$ & $\begin{array}{l}\text { Avance } \\
\text { significativo }\end{array}$ \\
\hline & $\begin{array}{l}\text { 2. El proyecto curricular es pertinente e inclusivo, brinda respuesta } \\
\text { a las demandas con relación al desempeño estudiantil fortaleciendo } \\
\text { el proceso de enseñanza-aprendizaje }\end{array}$ & Poco avance \\
\hline & $\begin{array}{l}\text { 3. La institución educativa cuenta con el sistema de liderazgo par- } \\
\text { ticipativo, alineando acciones, articulando funciones en una visión } \\
\text { común para fortalecer el proceso de enseñanza-aprendizaje. }\end{array}$ & $\begin{array}{l}\text { Avance } \\
\text { significativo }\end{array}$ \\
\hline \multirow{3}{*}{ 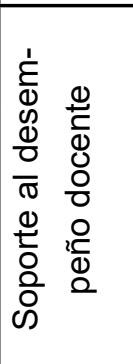 } & $\begin{array}{l}\text { 4. Cuenta con un equipo de docentes preparados y con un plan de } \\
\text { desarrollo profesional. }\end{array}$ & Poco avance \\
\hline & $\begin{array}{l}\text { 5. Implementa procesos que garanticen que el proyecto curricular } \\
\text { institucional se concretice en programaciones curriculares perti- } \\
\text { nentes según las necesidades estudiantiles. }\end{array}$ & $\begin{array}{l}\text { Avance } \\
\text { significativo }\end{array}$ \\
\hline & $\begin{array}{l}\text { 6. Las acciones pedagógicas aseguran el desarrollo de competen- } \\
\text { cias planificadas. }\end{array}$ & Poco avance \\
\hline
\end{tabular}




\begin{tabular}{|c|c|c|}
\hline \multirow[b]{2}{*}{ 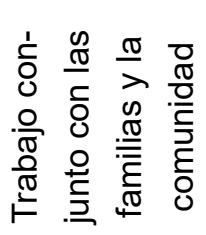 } & 7. Integra a las familias en el proceso de enseñanza aprendizaje. & Poco avance \\
\hline & $\begin{array}{l}\text { 8. Integra a las familias y a la comunidad en estrategias para forta- } \\
\text { lecer la formación de los estudiantes. }\end{array}$ & Poco avance \\
\hline \multirow{2}{*}{ 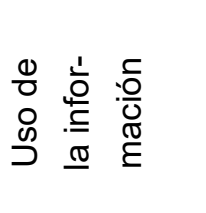 } & $\begin{array}{l}\text { 9. Procesa información relevante sobre progreso docente y estudi- } \\
\text { antil. }\end{array}$ & Poco avance \\
\hline & $\begin{array}{l}\text { 10. Diseña e implementan planes de mejora basados en la política } \\
\text { de priorización. }\end{array}$ & $\begin{array}{l}\text { Avance signifi- } \\
\text { cativo }\end{array}$ \\
\hline \multirow{2}{*}{ 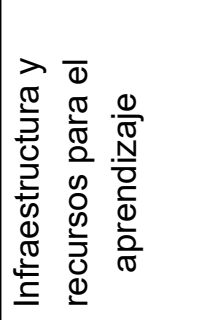 } & $\begin{array}{l}\text { 11. Monitorea la gestión de infraestructura tanto a nivel de manten- } \\
\text { imiento como a adquisición de nuevas instalaciones. }\end{array}$ & Poco avance \\
\hline & $\begin{array}{l}\text { 12. La gestión de recursos se desarrolla de manera transparente y } \\
\text { orientada a mejorar la enseñanza aprendizaje. }\end{array}$ & Poco avance \\
\hline
\end{tabular}

Resultados de encuesta y ficha de análisis documental

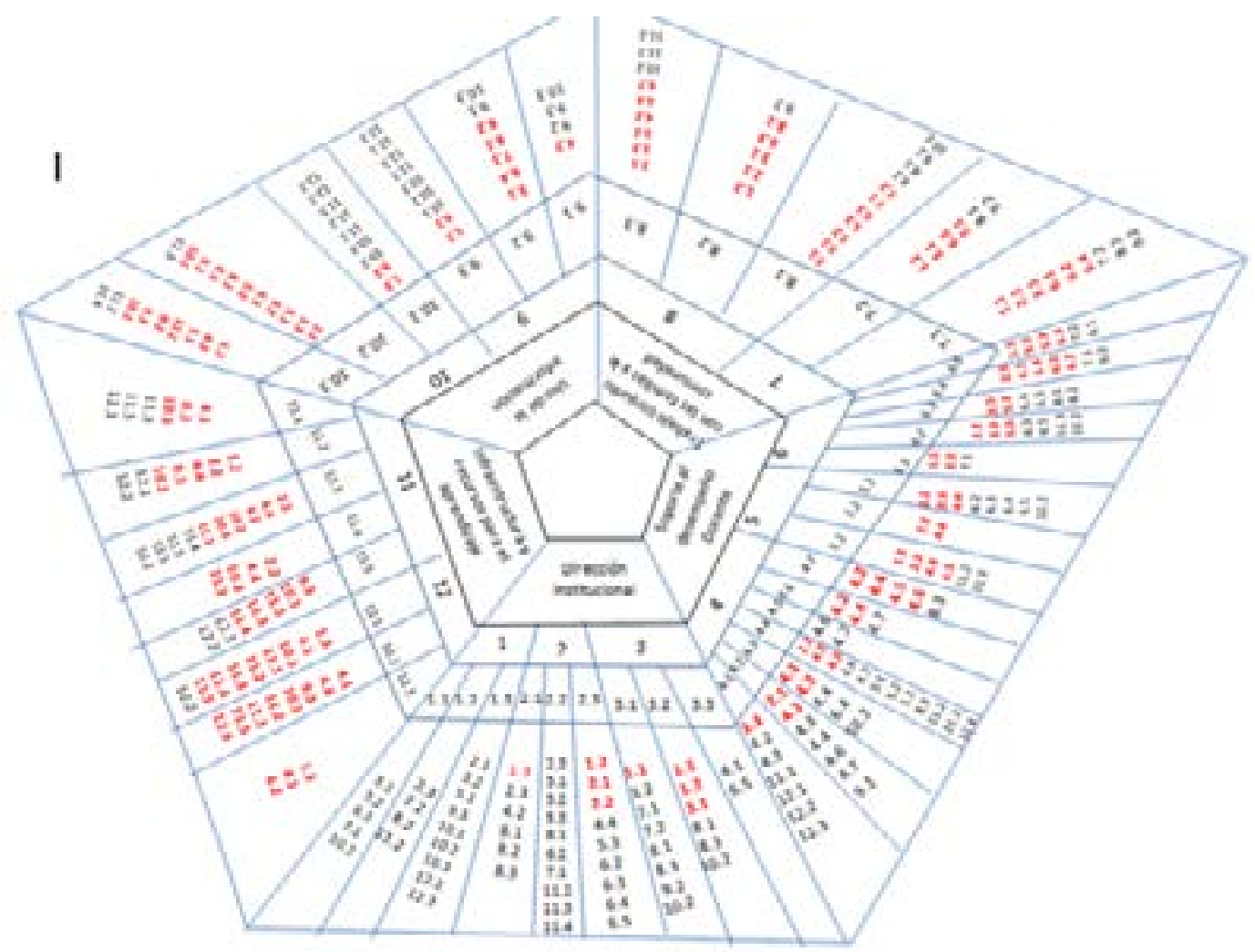

Figura 3. Resultados sistematizados en el pentágono relacional Elaboración propia. 
Se utilizará esta misma tabla para establecer el nivel de avance de estándares, factores y dimensiones.

\section{Tabla 2}

Niveles de calificación de factor, estándar

\begin{tabular}{|l|c|}
\hline Nivel de avance & Puntajes \\
\hline En inicio & {$[1,0-1,5>$} \\
\hline Poco avance & {$[1,5-3,0>$} \\
\hline Avance significativo & {$[3,0-3,7>$} \\
\hline Logrado & {$[3,7-4,0]$} \\
\hline
\end{tabular}

Tomado de modelo Sineace

\section{DISCUSIÓN DE RESULTADOS}

En la autoevaluación institucional, se asume la organización como unidad básica de cambio, impulsa evoluciones sinérgicos; para ello, lleva a la comunidad educativa a reflexionar sobre si los quehaceres diarios se encuentran alineados con la integralidad de la formación estudiantil. Esta investigación busca promover el proceso de autoevaluación institucional con fines de acreditación por medio de ello, recoger información de manera sistemática de los estados de los estándares de la gestión institucional, con la finalidad de hacer un reconocimiento oportuno de las fortalezas, oportunidades, debilidades y amenazas. Los resultados han demostrado que el nivel de relación entre los factores y estándares es directo debido a que el desarrollo o retraso de los indicadores de estándares mengua o impulsa al factor por ser dimensión incluyente. Como dice Villanueva (2005, en Jiménez A.) tanto la autoevaluación como la acreditación se han convertido en herramientas eficaces para la mejora de la calidad de la educación.

Así, los hallazgos en la presente investigación son los siguientes:

En el estándar 1, orientado a verificar la participación en la construcción del proyecto educativo enfocado en la mejora del proceso de enseñanza-aprendizaje de manera pertinente, inclusiva, se encuentra en un nivel de 3.11. Significa que es avance significativo, el indicador más alto de este estándar tiene como puntaje 3.75 , que corresponde comprender que la visión en el proyecto educativo institucional debe considerar las características y situaciones significativas de los estudiantes y comunidad. Asimimo, el indicador más bajo tiene como puntaje 2.57 , que corresponde a alinear la misión, visión, valores y objetivos estratégicos de manera inclusiva coadyuvando a desarrollar las capacidades en las áreas curriculares y las competencias. Entendemos que el proyecto curricular es de idóneo cuando se adecua a las necesidades estudiantiles. La Unesco en Stabback Philip (2016) manifiesta que debemos implementar una educación inclusiva con calidad y donde todos sin excepción tengan oportunidades de aprendizaje, donde se desarrollen conocimientos, capacidades, valores, habilidades y competencias conexas, buscando alinear las aspiraciones políticas de la educación en el currículo caso contrario no será posible garantizar educación de calidad.

En cuanto al estándar 2, orientado a verificar si el proyecto curricular es pertinente e inclusivo dando respuesta a las demandas con relación al desempeño estudiantil fortaleciendo el proceso de enseñanza-aprendizaje, se encuentra en un nivel de 2.39. Significa que está en poco avance, cabe resaltar que el indicador más bajo con 2.00 corresponde a contar con un proyecto curricular como base para la aplicación de estrategias pedagógicas correspondiente a la diversidad estudiantil y evidencia que está en un nivel de poco avance. Si consideramos a la escuela como ese pequeño piloto de la sociedad encontramos que reproduce las desigualdades de afuera, así como las oportunidades. De ahí que el proyecto curricular deberá ser tan versátil como la variedad de nuestros estudiantes, tan inclusivo que satisfaga los interese colectivos a partir del intercambio de las diferencias, tan 
nutrido de estrategias didácticas pertinentes que sea tácitamente factible la recuperación de experiencias de aprendizaje ya sea por asociación por similitud, contraste, contigüidad o por recuperación inusitada, tan político que recoja la intención educativa a partir de la participación democrática y ciudadana de la comunidad y otros organismos vinculantes.

El estándar 3, orientado a verificar si la institución educativa cuenta con el sistema de liderazgo participativo alineando acciones, articulando funciones en una visión común para fortalecer el proceso de enseñanza-aprendizaje, se encuentra en un nivel de 3.00 de puntaje, que significa que está en poco avance. Es necesario resaltar que el indicador más alto de este estándar es 3.75 rango insuficiente para demostrar logramos este estándar. Si consideramos las palabras del filósofo austriaco Feyerabend, «Una democracia es una asamblea de hombres maduros y no un rebaño de ovejas», nos ratificamos en la idea que existe la necesidad de democratizar las instituciones educativas incrementando el liderazgo participativo a través de roles y funciones de manera sostenible construyendo el liderazgo participativo.

El estándar 4 , orientado a verificar si contamos con un equipo de docentes preparados y con un plan de desarrollo profesional, tiene como puntaje 2.00, lo cual implica que está en poco avance. Cabe precisar que hay dos indicadores que están bajos y corresponden a la etapa de en inicio que refieren a la poica gestión que se viene haciendo para brindar capacitación sostenida a los docentes según sus necesidades, el cual tiene puntaje 1.00. Tampoco hemos desarrollado la cultura de compartir experiencias con otras instituciones que servirían de para enriquecer las capacidades docentes a partir de la recreación de casos y escenarios distintos a lo acostumbrado; en este indicador, también hay puntaje 1.00, es decir, en inicio. Según Miranda (2018), nos dice que existe poca confianza en los docentes sobre su práctica pedagógica muy a pesar de sus certificaciones equivalentes a la de otros países y años de experiencia; eso se puede ver por el proceso de formación profesional acompañado de las políticas de desarrollo docente. Como se dijo en Portocarrero-Sierra (2020), la inversión en el capital humano en educación involucra desarrollar conocimiento, formación a las personas lo que lleva a un mejor rendimiento e involucramiento productivo en la economía moderna.

El estándar 5 , orientado a verificar si implementamos procesos que garanticen que el proyecto curricular institucional se concretice en programaciones curriculares pertinentes según las necesidades estudiantiles, tiene como puntaje 3.44. Esto implica que está en la etapa de avance significativo, considerando que el indicador referido a asegurar que las programaciones curriculares conduzcan a desarrollar las competencias en todas las áreas tiene un puntaje 4.00, aparece como logrado, se deduce que debemos continuar laborando en función a las programaciones curriculares. Aseguramos que la programación curricular de cada grado/ciclo y área esté alineada con las programaciones curriculares de los otros grados/ciclos y áreas, tiene un puntaje de 3.33 , avance significativo. Cabe mencionar también que este estándar es el más alto que ha logrado la Institución Educativa, con 3.44 , el cual es de avance significativo. Según Pérez (2011) y Ruiz en Saldarriaga (2016), las actividades pedagógicas deben garantizar la acción de los agentes educativos en todas las etapas del diseño de la propuesta curricular, y es la única manera de ser concretada y anticipada bajo esta modalidad. Saldarriaga (2016) indica que el diseño curricular nacional encuentra su concreción en la diversificación curricular, y da respuesta a sus particularidades provenientes del multiculturalismo y multilingüismo; además, considera los filtros provenientes de las instancias de planificación curricular y la transversali- 
zación; así como la intervención de otros agentes.

El estándar 6, orientado a verificar si las acciones pedagógicas aseguran el desarrollo de competencias planificadas, tiene como puntaje 2.20; es decir se encuentra en una etapa de poco avance, el indicador más bajo y se traduce a raíz de la poca capacidad de desarrollar actividades y estrategias de aprendizaje planificadas por cada área curricular, donde tiene un puntaje de 1.00; lo que demuestra que está en inicio. La educación no es ajena a los cambios sociales y entre los más determinantes es indefectiblemente la construcción de la sociedad de conocimiento que basa su revolución en el uso de tecnologías de información y comunicación. Esta, según Salguero, F.L. y J.R. Peña en Martínez Olga (2018), nos dice su acercamiento como recursos didáctico permite al estudiante aprender temáticas diversas, así como adiestrarse en el uso de tics como mediadores de aprendizaje que siempre se encuentra renovando; en ese sentido, el uso de las tecnologías de información y comunicación presentan una gama de oportunidades didácticas para la adecuación pertinente al proceso de aprendizaje.

El estándar 7 , orientado a verificar si integramos a las familias en el proceso de enseñanza-aprendizaje, tiene un puntaje de 2,50; es decir se encuentra en una etapa de poco avance. El indicador que involucra a la familia en el análisis de los estudiantes para implementar estrategias de aprendizaje es el más bajo puntaje de 2.00 y está en un nivel de poco avance. Lo cual lleva a la siguiente reflexión: según Alicia Razeto (2016), de cuatro aspectos que involucran a la familia debemos considerar que estas inciden en el desempeño de los estudiantes. La condición de vulnerabilidad familiar genera menores condiciones de apoyo educativo a los estudiantes, incluso recorta las oportunidades de relacionarse con la institución educativa. Por más que el Estado destaque la importancia de involucrar a las familias, hasta ahora no se ven resultados óptimos mostrando insuficiencia; además, es importante considera experiencias de otros países para tener una línea referencial como base para enriquecer el vínculo familia institución educativa.

El estándar 8, orientado a verificar si integramos a las familias y a la comunidad en estrategias para fortalecer la formación de los estudiantes, tiene un puntaje de 2.00; es decir, está en un nivel de poco avance. El indicador donde las familias se incorporan con sus saberes y experiencias en el desarrollo de competencias en los estudiantes es el más bajo de este estándar con un puntaje de 1.00 , y está en un nivel de en inicio; mientras que el indicador que busca integrar a las instituciones para generar recursos orientadas a fortalecer el aprendizaje es el más alto de este estándar con un puntaje de 4.00, y está en un nivel de logrado. Esta situación a modo de decir, según Casassus (1999), es importante orquestar nuevos actores sociales en la tarea educativa, comunidades locales, sector productivo, político y otros. Otro aspecto importante hoy en día es que podemos encontrar el amparo en el Proyecto Educativo Nacional al 2036, que tiene por condición ser multisectorial, en otras palabras, compromete la planificación de todos los agentes de la estructura del estado peruano, dado que impulsan el proyecto Perú. De ahí que deviene la importancia de incorporar a actores comunitarios no solo, con el fin de recopilar ayuda, sino porque ellos son los configuradores del sistema que finalmente recibirán a los estudiantes.

El estándar 9, orientado a verificar si procesamos información relevante sobre progreso docente y estudiantil, tiene un puntaje de 2.28; es decir, está en un nivel de poco avance. El indicador que busca la participación de todos los miembros de la comunidad educativa en la evaluación tiene un puntaje de 1.44; es decir, está en un nivel de poco avance, el cual es el 
más bajo de los indicadores. Es determinante la importancia del trabajo colegiado en las instituciones educativas, ya que propicia el intercambio de experiencias basadas en la identificación de casos específicos y la elaboración sustantiva de mejoras en los procesos de aprendizaje, a partir de la evaluación de la realidad estudiantil con la participación education peers del equipo docente y otros miembros de la comunidad educativa.

El estándar 10 , orientado a verificar si diseñamos e implementamos planes de mejora basados en la política de priorización, tiene un puntaje de 3.21, es decir, avance significativo. Asimismo, observamos que el indicador de priorización, seguimiento e implementación de planes de mejora se encuentra en un estado de poco avance, con un puntaje de 1.62. Según Rivera et al (2015), existen cinco factores que determinan la eficacia de los planes de mejora; entre ellos, tenemos el desarrollo curricular y la organización institucional, aspectos estrictamente personales, habilidades profesionales, dinámicas de intercambio y convivencia involucrados en el desempeño de los recursos humanos. Todos ellos respaldados por los recursos suficientes para su ejercicio, de ahí que, según Bolívar (2015), para batir estos elementos, es importante que los centros escolares se configuren como comunidades de aprendizaje de los estudiantes y docentes, que la planificación y desarrollo de planes de mejora sea sostenida y autoevaluada de manera permanente.

El estándar 11, orientado a verificar si hacemos seguimiento a la gestión de infraestructura tanto a nivel de mantenimiento como a adquisición de nuevas instalaciones, tiene un puntaje de 1.00; es decir, se encuentra recién en inicio. Situación que evidencia la necesidad de evaluar los procesos de manera recurrente y permanente, que afronte la situación de precariedad académica por infraestructura inadecuada que no permite optimizar el desempeño docente que finalmente minimiza la calidad de la formación en educación básica regular. En Perú se ha iniciado el Plan Nacional de Infraestructura Educativa al 2025 que busca mejorar las condiciones, la capacidad, la gestión y la sostenibilidad en las instalaciones educativas. Este tiene cuatro objetivos estratégicos que buscan condiciones de seguridad y funcionalidad en instalaciones existentes, cubrir brecha de infraestructura no existente, mejorar la gestión de infraestructura, hacer sostenible la infraestructura educativa existente. Su propósito tiene como meta el año 2025, con muchas necesidades y expectativas, se espera el cumplimiento de este plan para lograr superar esta limitación determinante en la provisión de servicios educativos de calidad.

El estándar 12, orientado a verificar si la gestión de recursos se desarrolla de manera transparente y orientada a mejorar la enseñanza-aprendizaje, se encuentra en un nivel de 2.19. Asimismo, se resalta que el indicador más bajo de este estándar en condición de poco avance con 1.58 de puntaje, y se refiere a la comunicación oportuna de la rendición de cuentas tanto de financiamiento como de cumplimiento de metas de logros de aprendizaje y planes de mejora manera periódica. Tal como manifiesta Salazar M (2019), la transparencia fortalece el vínculo entre los servidores y la ciudadanía, lo que incrementa la confianza en la institución educativa, facilita mayor participación a la comunidad educativa en la gestión de la institución y previene la corrupción, ya que se encuentra bajo el principio de vigilancia ciudadana, habilita otros derechos como opinión pública, voto informado, libertad de expresión y otros, incrementa la democracia y eficiencia.

Resulta útil precisar en cada estándar, factor e indicador de desarrollo institucional, dando luces para planificar las mejoras de manera integral o sistémica; además, los factores que se han considerado son pertinentes al con- 
texto de la Educación Básica Regular, lo que posibilita su aplicación a otras instituciones del mismo nivel.

\section{CONCLUSIONES}

Se puede concluir que el proceso de autoevaluación en la institución educativa Santo Toribio de Mogrovejo que abordó cinco factores en los niveles de logro alcanzados en mayoría están en nivel poco avance. Esto indica que es necesario priorizar las atenciones considerando la relación más directa con el proceso de enseñanza- aprendizaje y la formación integral de los estudiantes.

Los factores que se encuentran en nivel de poco avance son los siguientes: Dirección institucional en el estándar a verificar si el proyecto curricular es pertinente e inclusivo, respondiendo a las demandas con relación al desempeño estudiantil, fortaleciendo el proceso de enseñanza-aprendizaje. Soporte al desempeño docente, en el estándar, vinculado a las acciones pedagógicas aseguran el desarrollo de competencias planificadas. Finalmente, el factor Participación conjunta con las familias y la comunidad, en el estándar que integra las familias y a la comunidad en estrategias para fortalecer la formación de los estudiantes; en el factor Infraestructura y recursos para el aprendizaje en el estándar hacemos seguimiento a la gestión de infraestructura, tanto a nivel de mantenimiento como a adquisición de nuevas instalaciones y la gestión de recursos se desarrolla de manera transparente y orientada a mejorar la enseñanza-aprendizaje.

Dado que se trata de una investigación participativa donde la comunidad educativa se ha visto involucrada en el descubrimiento de las fortalezas y las limitaciones, se ha incrementado el compromiso de los miembros de la comunidad educativa en la planificación y la disposición a la implementación de los planes de mejora institucional a partir de la reflexión y discusión.
El modelo de autoevaluación promovido por el Sistema Nacional de Evaluación, Acreditación y Certificación de la Calidad Educativa es útil para monitorear la institución de manera sistemática. Por ello, se sugiere generar nuevas investigaciones de esta naturaleza en otras instituciones de Educación Básica Regular con los mismos factores, estándares e indicadores promovidos por el marco legal del sistema educativo peruano.

\section{REFERENCIAS BIBLIOGRÁFICAS}

Acuerdo Nacional para Trabajar de Acuerdo. (2006). Políticas de Estado y planes de gobierno. Recuperado de http:// acuerdonacional.pe/wp-content/ uploads/2014/06/PoliticasEstado PlanesGobierno.pdf

Aguila Salazar, M. (2019). Aspectos generales en materia de Transparencia y Acceso a la Información Pública Ministerio de Justicia y Derechos Humanos Autoridad Nacional de Acceso a la Información Pública. Recuperado en https://www.minjus. gob.pe/wp-content/uploads/2020/10/ PROCEDIMIENTO-DE-ATENCIONSAIP-MARCIA-AGUILA.pdf

Alaiz, V., Góis, E., e Gonçalves, C. (2003). Autoavaliação de escolas. Pensar e praticar. Porto: Edições ASA.

Banco Mundial, Ministerio de Educación del Perú (2016). Infraestructura educativa al 2025. Colecciones Informes técnicos. Recuperado de http://documents 1. worldbank.org/curated/ en/995001476430088084/pdf/109095BRI-P160939-Series-Per\%C3\%BA-Notas-de-Pol\%C3\%ADtica-2016-PUBLICInfraestructuraeducativaal.pdfBolívar, A. (2014). Un liderazgo pedagógico en una comunidad que aprende. Revista Padres y Maestros, (361), 23-27. DOI: https://doi. org/10.14422/pym.i361.y2015.004 
Casassus, J. (1999). Conceptuales para el análisis de los cambios en la gestión de los sistemas educativos. En Unesco (Ed.), La gestión en busca de sujeto, Santiago de Chile.

Consejo Nacional de Educación (2020). Proyecto Educativo Nacional al 2036. Ministerio de Educación. Recuperado de https://www. cne.gob.pe/uploads/publicaciones/2020/ proyecto-educativo-nacional-al-2036.pdf

Decreto Supremo N. ${ }^{\circ}$ 018-2007-ED. Aprueban Reglamento de la Ley N..$^{\circ} 28740$, Ley del Sistema Nacional de Evaluación, Acreditación y Certificación de la Calidad Educativa. (2007). Recuperado de https:// www.gob.pe/institucion/minedu/normaslegales/118163-018-2007-ed

De la Cruz Flores, G. (2016). Justicia Curricular: Significados e Implicaciones. Revista Electrónica de Educación Sinéctica, (46). Recuperado en https://www.redalyc.org/ jatsRepo/998/99843455010/html/index. html

Gutiérrez-Anguiano, N., y Chaparro CasoLópez, A. (2020). Evidencias de fiabilidad y validez de una escala para la autoevaluación de las prácticas de enseñanza en secundaria. Perfiles educativos, 42(167). Recuperado en http://perfileseducativos. unam.mx/iisue_pe/index.php/perfiles/article/view/59193Feyerabend, P. (1985). ¿Por qué no Platón? Madrid: Editorial Tecnos.

Ipeba, Ministerio de Educación del Perú (2013). ¿Qué y cómo evaluamos la gestión de la institución educativa? Recuperado en https://www.sineace.gob.pe/wpcontent/uploads/2014/08/GUIA EBR ccarat.pdf

Jimenez Romero, J. (2019). The evaluation and accreditation of professional education in Mexico: legitimacy and competitiveness as the goal of the university? Rev. educ. sup [online], 48(189), pp. 55-72. Recuperado de http://www. scielo.org. $\mathrm{mx} / \mathrm{scielo}$.php?pid=S0185$27602019000100055 \&$ script $=$ sci abstract\&tIng=en

Martínez, O., Steffens, E., Decired, O., y Hernández, H. (2018). Estrategias Pedagógicas Aplicadas a la Educación con Mediación Virtual para la Generación del Conocimiento Global. Formación universitaria, 11(5). Recuperado de http://dx.doi.org/10.4067/ S0718-50062018000500011

Ministerio de Educación (2020, 26 de febrero). Evaluación Censal, Reporte Nacional 2019. Evaluaciones nacionales de logros de aprendizaje [artículo web]. http://umc.minedu.gob.pe/resultadosnacionales2019/

Miranda Jaña, C. (2018). Formación Permanente e Innovación En Las Prácticas Pedagógicas en Docentes de Educación Básica. Estudios Pedagógicos, 31(1), pp. 63-78. DOI:10.4067/S071807052005000100004

Leite, C., y Sampaio, M. (2020). Autoevaluación y justicia social en la evaluación de las escuelas en portugal. Cad. Pesqui, 50(177). http://dx.doi. org/10.1590/198053146835

Ley N. ${ }^{\circ}$ 28044. Ley General de Educación. (2003). https://www.gob.pe/institucion/ congreso-de-la-republica/normas-legales/118378-28044 
Ley $\mathrm{N}^{\circ}$ 2947-2017-PE. Ley de la evaluación acreditación y certificación de la calidad educativa. (2016). https://leyes.congreso.gob.pe/Documentos/2016 2021/Proyectos de Ley $y$ de Resoluciones Legislativas/ PL0294720180604..pdf

Palma, J. B. (2001). O papel das diferentes modalidades de avaliação das escolas na regulação das políticas educativas. $A d-$ ministração Educacional, 1, pp. 36-40.

Paz, S., y Torchiani, R. (2020). Un modelo de acreditación que asegure la mejora de la calidad de un programa de estudios. Experiencia en el nivel universitario. Publicaciones, 50(4), 141-156. Recuperado de

https://digibug.ugr.es/bitstream/ handle/10481/66145/17787-51962-1PB.pdf?quence $=1$ \&isAllowed $=y$

Presidencia del Consejo de Ministros. (2019). Política Nacional de Competitividad y productividad [artículo web]. https://portal.concytec.gob.pe/index.php/politicas/ item/265-politica-nacional-de-competitividad-y-productividad-2019-2030

Portocarrero L. et al (2020). Evaluación del impacto de la acreditación de alta calidad en las instituciones públicas de educación superior de Colombia, Formación Universitaria, 13(6). Recuperado de http://dx.doi.org/10.4067/S0718$\underline{50062020000600037}$

Razeto, A. (2016). El involucramiento de las familias en la educación de los niños. Cuatro reflexiones para fortalecer la relación entre familias y escuelas. Páginas de Educación, 9(2). Recuperado de http://www. scielo.edu.uy/scielo. php?pid=S168874682016000200007\&script=sci_abstract

Rivera Núñez, C., y Volante Beach, P. (2015). Teorías subjetivas de docentes directivos sobre factores que definen la eficacia de los planes de mejoramiento educativo ley SEP. Estudios pedagógicos, 41(1). Recuperado de http://dx.doi.org/10.4067/ S0718-07052015000100014

Saldarriaga, Y. (2016). Participación docente en el diseño del proyecto curricular institucional de una institución educativa pública de San Juan de Lurigancho [tesis para obtener el grado de magíster]. Pontificia Universidad Católica. Recuperado en https://search.proquest.com/ openview/2fdda1c60403690694765b32 8def10d6/1?pq-origsite=gscholar\&cbl=1 $8750 \&$ diss $=y$

Sousa, A., y Terrasêca, M. (2015). Processos de autoavaliação de escolas ou avaliação interna do sistema? Arquivos Analíticos de Políticas Educativas, 23(115), 1-15. Recuperado de https://www.redalyc.org/ pdf/2750/275041389106.pdf

Stabback, P. (2016). Qué hace a un currículo de calidad. Cuestiones fundamentales y actuales del currículo $y$ el aprendizaje. Reflexiones en progreso N. ${ }^{\circ} 2$. Unesco. Recuperado de http://repositorio.minedu.gob.pe/bitstream/handle/20.500.12799/4256/ Qu\%c3\%a9\%20hace\%20a\%20un\%20 curr \%c3\%adculo\%20de\%20calidad. pdf?sequence $=1 \&$ isAllowed $=y$ 
Salguero, F., y Peña, J. (2015). The ICT Coordination and Teacher Education as Drivers of Educational Reform in the School, Enseñanza y Teaching, 33(2). Recuperado en https://dialnet.unirioja.es/servlet/ articulo?codigo $=5581206$

Vezub, L. (2007). La formación y el desarrollo profesional docente frente a los nuevos desafíos de la escolaridad Profesorado. Revista de Currículum y Formación de Profesorado, 11(1), 1-23. Recuperado de https://www.redalyc.org/ pdf/567/56711102.pdf 